\title{
GPU-BASED VOLUME RECONSTRUCTION FROM VERY FEW ARBITRARILY ALIGNED X-RAY IMAGES*
}

\author{
DANIEL GROSS ${ }^{\dagger}, \mathrm{ULRICH}_{\text {HEIL }}^{\dagger}$, RALF SCHULZE ${ }^{\ddagger}$, ELMAR SCHOEMER $^{\dagger}$, AND \\ ULRICH SCHWANECKE§
}

\begin{abstract}
This paper presents a three-dimensional GPU-accelerated algebraic reconstruction method in a few-projection cone-beam setting with arbitrary acquisition geometry. To achieve artifact-reduced reconstructions in the challenging case of unconstrained geometry and extremely limited input data, we use linear methods and an artifact-avoiding projection algorithm to provide high reconstruction quality. We apply the conjugate gradient method in the linear case of Tikhonov regularization and the two-point-step-size gradient method in the nonlinear case of total variation regularization to solve the system of equations. By taking advantage of modern graphics hardware we achieve acceleration of up to two orders of magnitude over classical CPU implementations.
\end{abstract}

Key words. image reconstruction, X-ray, cone-beam tomography, ART, GPU, arbitrary geometry, few-view reconstruction, conjugate gradient method, regularization

AMS subject classifications. Primary, 92C55, 65F22; Secondary, 92C50, 65R32, 65N21

DOI. $10.1137 / 080736739$

1. Introduction. Tomographic reconstructions of a three-dimensional (3D) object from its projection images are becoming more and more widespread. Depending on the projection imaging modality, miscellaneous methods are used to achieve satisfactory reconstruction results within an acceptable time frame. Most common reconstruction methods in standard computed tomography are the filtered backprojection method based on the Radon inversion formula. In many application areas 3D information is desired, although only a severely limited amount of data can be collected. In intraoral imaging or mammograms, for instance, measurements can be obtained only in a very small angular field [26, 24, 10, 11, 18, 27, 30]. Industrial applications such as systems for material examination or luggage inspection usually are made up of an assembly containing only a few projection units in a restricted projection geometry [12]. The limited amount of collectible data, based on geometric restrictions or the maximum admissible radiation exposure, leads to unsatisfactory results when well-established reconstruction methods such as the filtered backprojection are applied [13].

In this paper, we focus on the reconstruction of 3D objects from an extremely limited amount of cone-beam projection images (approx. 5 to 30) obtained in arbitrary geometry. On the one hand arbitrary geometry means that there are no restrictions to the geometrical connection of the point-shaped X-ray source and the imaging plane. This especially permits large cone angles normally leading to severe reconstruction artifacts (see, e.g., [13]). One the other hand the position and orientation between the different images can be arbitrary in our setting. This means that the images do

\footnotetext{
* Received by the editors September 23, 2008; accepted for publication (in revised form) July 13, 2009; published electronically November 25, 2009.

http://www.siam.org/journals/sisc/31-6/73673.html

${ }^{\dagger}$ Institute of Computer Science, Johannes Gutenberg-Universität Mainz, 55099 Mainz, Germany (grossdaniel@web.de, ulrichheil@web.de, schoemer@informatik.uni-mainz.de).

${ }^{\ddagger}$ Department of Oral Surgery (and Oral Radiology), University Medical Center of the Johannes Gutenberg-University Mainz, 55131 Mainz, Germany (rschulze@mail.uni-mainz.de).

$\S$ Department of Design, Computer Science and Media, Hochschule RheinMain, University of Applied Sciences, Wiesbaden Ruesselsheim Geisenheim, Germany (ulrich.schwanecke@hs-rm.de).
} 


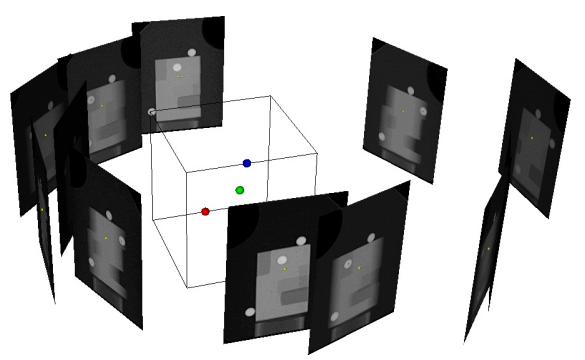

(a)

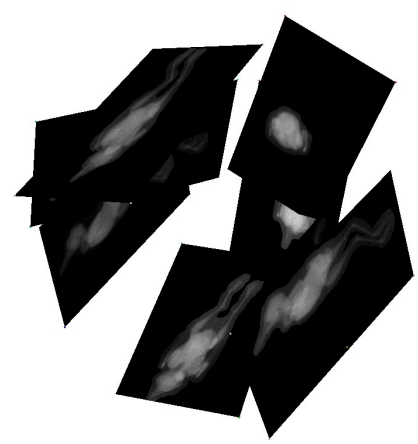

(b)

Fig. 1.1. Different arrangements of an X-ray system with arbitrary geometry. (a) Circular projection arrangement with varying focus-detector and object-detector distance as well as arbitrary rotation angles; (b) completely arbitrary configuration within eight degrees of freedom (see [21]). The reconstructions from these settings are displayed in Figures 5.5 and 5.6.

not need to be arranged in a circular setting nor aligned to any axis (see Figure 1.1 for some examples of different arbitrary imaging geometries).

In general, 3D reconstruction from two-dimensional (2D) projections is an illposed problem [25]. Common approaches to obtaining adequate solutions anyhow include Tikhonov regularization or simply stopping the iterative reconstruction process after a few steps [25]. Even worse than the fact that the problem in general is ill-posed is the missing information due to the limited number of projections or the bounded angle of exposure. To overcome this problem a priori information on the object to be reconstructed can be used. Regularization using total variation or the Besov norm, respectively, are based on object characteristics like smoothness or compactness to generate desirable reconstruction results (see, e.g., [10, 20, 23]). An eligible framework for obtaining 3D reconstructions in a setting with arbitrary imaging geometry and very few projections is given by the algebraic reconstruction techniques (ART) $[16,9]$. For few-view reconstructions, ART-based algorithms are well known to produce much better results than algorithms based on Radon transformation $[9,13]$. The fundamental idea of ART is to remodel the reconstruction problem into a proper linear system of equations. Due to its large size the obtained linear system is solved in an iterative way [9]. In recent years many parallel algorithms have been developed to solve the system within an acceptable time frame [13, 15, 7, 21].

The fundament of all algebraic reconstruction techniques is a robust algorithm for the projection and backprojection step, respectively. The projection step models the $\mathrm{X}$-ray transform, i.e., the projection of a volume into a detector plane, whereas the backprojection step describes the projection of the intensities recorded in the detector plane into the volume. The projection step approximates line integrals of the volume along X-ray beams. Design of a projection algorithm requires taking into account two important points. First, notice that projection and backprojection require about $99 \%$ of the overall computing time of the reconstruction process. Second, simple and therefore fast implementations of these operations lead to serious artifacts in the reconstructed volumes. Combined with arbitrary geometries and sparse information due to the very limited amount of projection images, these artifacts can completely corrupt the 3D reconstructions. Based on De Man and Basu's idea of using the area of overlap between pixels and voxels as an interpolation kernel [3], we developed a 
novel generalized and artifact-minimizing method suitable for arbitrary geometries.

In this paper we present an artifact-reducing projection algorithm designed for the particular setting of $3 \mathrm{D}$ reconstruction from very few $(\leq 30)$ projection images acquired in arbitrary geometry. The algorithm can be implemented very efficiently on modern graphics processing units (GPUs). Driven by the necessity of realistic real-time visualization in computer games, an incredible performance growth of the GPUs emerged. General purpose GPU, or GPGPU, has become a popular term for using GPUs for purposes other than computer games, e.g., for scientific computing. Due to their massively parallelized architecture, programmable GPUs lend themselves optimally to projection and backprojection operations [14, 17]. Proper usage of the parallel architecture of a GPU results in a speedup of up to two orders of magnitude [14, 17, 22, 29]. We formulate the projection and backprojection step as one-step operators which can be outsourced to the GPU. Each of these operators is parallelized with respect to pixels and voxels, respectively, and is thereby considerably accelerated. Our encapsulated one-step operators can easily be integrated into current algebraic reconstruction techniques. We demonstrate the practicality of our operators on the basis of a system that uses the conjugate gradient method to solve the linear system.

The remainder of this paper is organized as follows: In section 2 we describe the new projection algorithm, whereas the integration of this algorithm into a framework for solving a system of equations is discussed in section 3. Section 4 presents relevant details concerning the implementation of our method on modern graphics hardware. After presenting results in section 5, we conclude and discuss future developments.

2. The projection algorithm. In this paper, we focus on $3 \mathrm{D}$ reconstructions from cone-beam data. For a fixed source $a$ a cone-beam transformation

$$
(D f)(a, \theta)=\int_{0}^{\infty} f(a+t \theta) d t, \quad a \in \mathbb{R}^{3}, \theta \in S^{2},
$$

is given by the line integral of the volume $f$ towards the direction $\theta$. As a parametrization $p: \mathbb{R}^{2} \rightarrow \mathbb{R}^{3}$ of the radiograph $g$ we choose

$$
p(x, y)=p_{0}+x p_{x}+y p_{y} \quad \text { with } \quad p_{x} \perp p_{y} \quad \text { and } \quad\left\|p_{x}\right\|=\left\|p_{y}\right\|=s,
$$

where the scaling factor $s \in \mathbb{R}$ represents the pixel size in the discretized model we describe beneath. Now the image intensities $g: \mathbb{R}^{2} \rightarrow \mathbb{R}$ can be evaluated by

$$
g(x, y)=\int_{L} f d t \quad \text { with } \quad L(t)=a+t \frac{p(x, y)-a}{\|p(x, y)-a\|} .
$$

For fixed source location $a$ and image plane $p$ this is the following special parametrization of the cone-beam transformed $D f$

$$
g(x, y)=(\Psi f)(x, y):=\int_{L} f d t=(D f)(a, \theta(x, y)) \quad \text { with } \quad \theta(x, y)=\frac{p(x, y)-a}{\|p(x, y)-a\|} .
$$

Discretizing the image $g$ by splitting it into $M=m^{2}$ square pixels as well as the volume $f$ using $N=n^{3}$ cubic voxels result in

$$
g=\sum_{i=1}^{M} g_{i} G_{i} \quad \text { and } \quad f=\sum_{j=1}^{N} f_{j} F_{j},
$$


where $g_{i}$ is the intensity, $G_{i}$ the characteristic function of the pixel area, and $f_{j}$ the intensity with $F_{j}$ as characteristic function of the voxel volume.

In the following we denote the set of indices of the voxels in the volume layer $k$ with $S_{k}$ and examine the projection $g^{k}=\Psi f^{k}$ of a single volume layer $f^{k}=\sum_{j \in S_{k}} f_{j} F_{j}$ onto an image $g^{k}$. The measurement of the line integral $g_{i}^{k}=\left(\Psi f^{k}\right)_{i}=\int_{L_{i}} f^{k} d s$ can be broken up into two parts. At first we have to compute the running length $d_{i}$ of ray $L_{i}$ within the volume layer $f^{k}$. The second part consists of extracting a sample value of this ray within the current slice. Common techniques obtain this value from simple nearest-neighbor sampling or bilinear interpolation. The projection method presented here computes this sample value through interpolation weights defined by the ratio of overlapping areas. Therefore we perspectively project the pixel area onto the midplane of a volume layer and define the interpolation weight that belongs to pixel $i$ and voxel $j$ as the overlap of the projected pixel area and the voxel area. Due to the fact that the ratio of pixel width and distance between pixel and radiation source in practice is very small, all rays from one pixel area to the source can be assumed to be parallel within the volume layer and thus in all volume layers. Hence the perspective projection of the pixel area can be considered as orthogonal.

If $o_{i}^{k}$ denotes the size of the backprojected pixel area of pixel $i$ onto the midplane of volume layer $k$ and $o_{i j}$ denotes the overlap of that area with voxel $j$, then the intensity $g_{i}^{k}$ of the pixel is given by the linear combination

$$
g_{i}^{k}=\sum_{j \in S_{k}} d_{i} \frac{o_{i j}}{o_{i}^{k}} f_{j}
$$

In a broader sense the term $w_{i j}^{k}=\frac{o_{i j}}{o_{i}^{k}}$ can be interpreted as an interpolation weight. But in contrast to other interpolation kernels this method is inherently avoiding aliasing artifacts due to the consideration of the size of pixels (and voxels) and hence also the distance of adjacent elements.

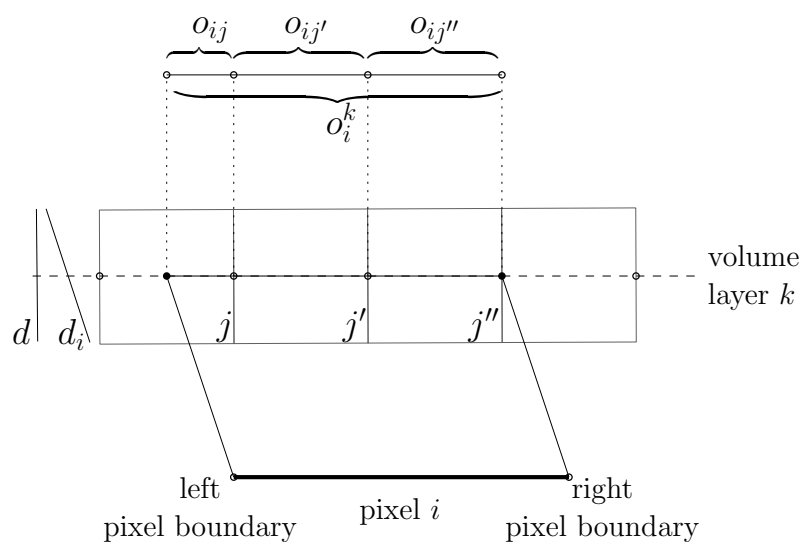

FIG. 2.1. Overlap measurement in 2D: overlap of two intervals (2D case).

In a two-dimensional setting $g_{i}^{k}$ can be evaluated by backprojecting the two pixel boundaries onto the midplane of the volume layer and iterating over all voxels lying between the projected boundaries (see Figure 2.1). This approach, termed distance driven by the authors, was introduced by De Man and Basu in [3]. For each voxel the area of overlap between two intervals has to be calculated. The generic overlap-based projection method is given in Algorithm 1. 


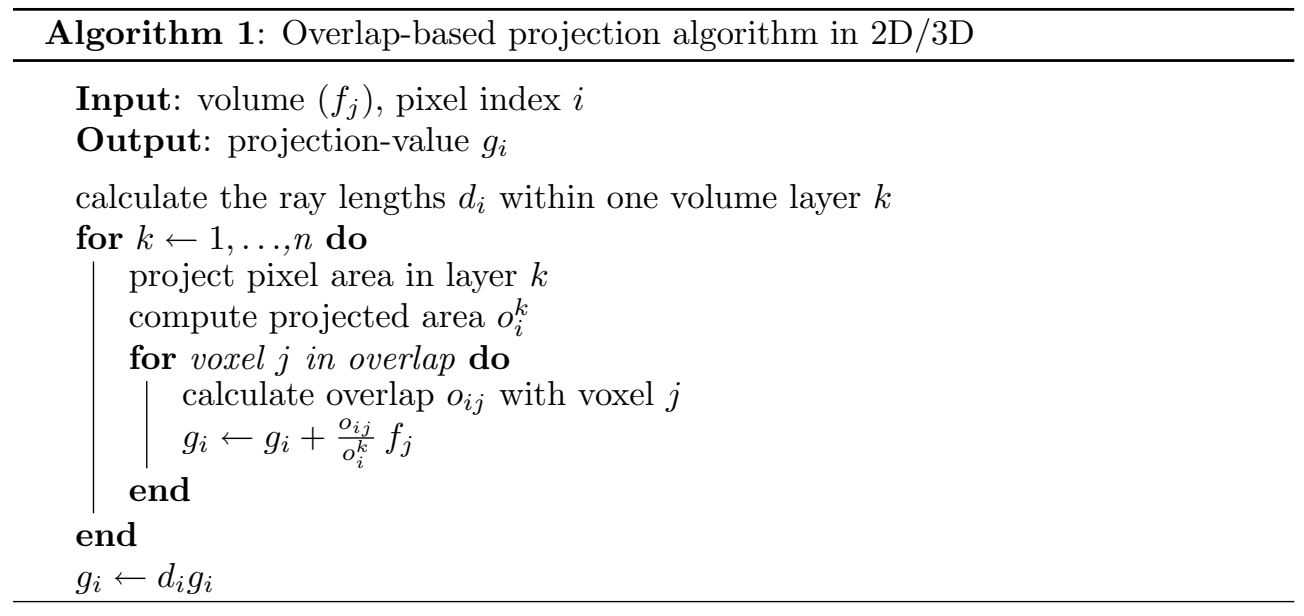

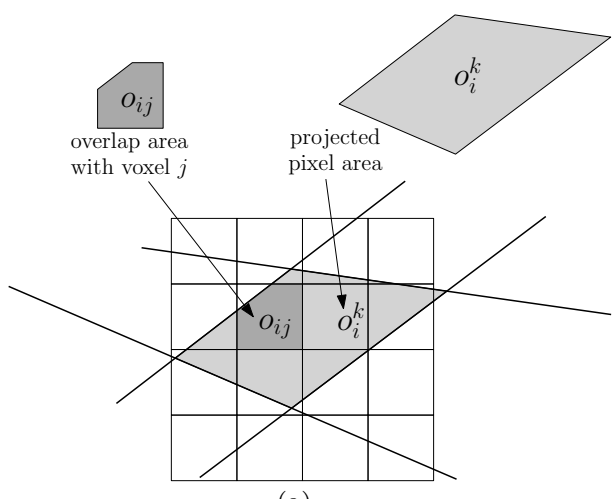

(a)

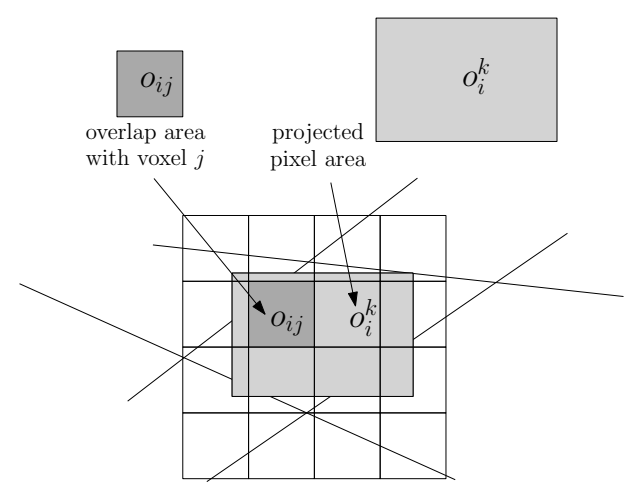

(b)

FIG. 2.2. Overlap measurement in the $3 D$ case: (a) exact overlap $o_{i j}$ of one pixel area $o_{i}^{k}$ (quadrangle) and the voxel grid exemplified by one voxel $j$; (b) overlap of approximated pixel and voxel grid.

To adopt the method to a 3D setting further simplifications have to be done in order to achieve acceptable operating times. In general the projective transformation of the pixel area is an arbitrary convex quadrangle. Calculating the intersections of a convex quadrangle with voxels is very time-consuming (see (a) in Figure 2.2). Therefore we substitute the quadrangles with rectangles aligned to the axes of the volume layer (see (b) in Figure 2.2). Recalling the very small ratio of the pixel width and the focus-pixel distance and the resulting small, pencil-like cone formed by tracing the pixel area back to the focus, one can show that a backprojection of a pixel into a volume layer forms approximately a parallelogram (see again Figure 2.2 (a)). If one (virtually) rotates the pixel area before the backprojection, the resulting parallelogram can be seen as $x$-axis aligned. In the following, an $x$-axis parallelogram can obviously be approximated by an axis-aligned rectangle. How this rectangle is exactly computed will be explained in the implementation part because it is closely related and fitted to the capabilities of the GPU. In contrast to the approach by De Man and Basu for three dimensions, we generalize the projective transformation between pixel and voxel completely, which is essential for arbitrary geometries. 
The overall discretization process defined by the projection equation (2.1) induces projection matrices

$$
A_{k}=D W_{k} \quad \text { with } D=\operatorname{diag}\left(d_{1}, \ldots, d_{N}\right) \text { and }\left(W_{k}\right)_{i j}=w_{i j}^{k}=\frac{o_{i j}}{o_{i}^{k}}
$$

mapping the volume layer $k$ into the image plane. Notice that $W_{k}$ can be interpreted as an adaptive interpolation kernel as $\sum_{j} w_{i j}^{k}=1$ holds. Furthermore the diagonal matrix $D$ containing the running length corrections for each single pixel does not depend on the volume layer. The backprojection matrix $A_{k}^{T}=W_{k}^{T} D$ maps a given image vector onto the volume layer $k$. It can be determined line by line in the same way as $A_{k}$ by mapping the area of a voxel $j$ into the image plane and calculating the overlapping area $\hat{o}_{j i}$ between this projection and the individual pixels, assumed to have size $s$. Again, the simplifying assumption is made that all rays crossing a fixed voxel are parallel to each other. With this in mind it does not matter if pixels are projected onto the midplane of a volume layer or if voxels are projected onto the image plane, because the ratio of areas is invariant under orthogonal projections. This assumption leads to

$$
\left(W_{k}\right)_{i j}=\frac{o_{i j}}{o_{i}^{k}} \approx \frac{\hat{o}_{j i}}{s^{2}}=:\left(\hat{W}_{k}\right)_{j i}
$$

As a result the complete projection matrix $A$ as well as the backprojection matrix $A^{T}$ for each image are given by

$$
A=D\left(\sum_{k} W_{k}\right) \quad \text { and } \quad A^{T} \approx\left(\sum_{k} \hat{W}_{k}\right) D
$$

The possibility of row-by-row access to the projection matrix as well as to the backprojection matrix is a fundamental precondition for the parallelization process for the GPU as detailed in section 4. This is due to the special read-write capabilities of the GPU, which require that one can explicitly compute one row of the backprojection matrix. The latter is not possible for the exact transpose. Consequently we have to approximate the transpose in an appropriate way. In contrast to ray-based methods our overlap-based approach yields a much better approximation of the transpose.

3. Solving the system of equations. In the following the projection matrix defined in (2.3), mapping a volume vector onto an image with number $l$, is denoted by $A^{l}=D^{l}\left(\sum_{k} W_{k}^{l}\right)$. Furthermore,

$$
A=\left[\begin{array}{c}
A^{1} \\
\vdots \\
A^{L}
\end{array}\right] \text { and } A^{T}=\left[\begin{array}{lll}
A^{1^{T}} & \ldots & A^{L^{T}}
\end{array}\right]
$$

refer to the complete projection matrix and its transposed, respectively.

We are looking for a solution of the typically underdetermined linear system $A x=b$, where the sparse matrix $A$ is given by (3.1). Thereby the vector $x$ represents the discretized densities of the volume $f$ and $b$ contains the given intensities of any image $g$ arranged in a single vector. Due to the additional influence of data and modeling errors, $A x=b$ is an ill-posed inverse problem in terms of Hadamard (see $[25,8]$ for further information on inverse problems). Because of the high dimension of the system only iterative solvers can be used. All iterative solvers commonly 


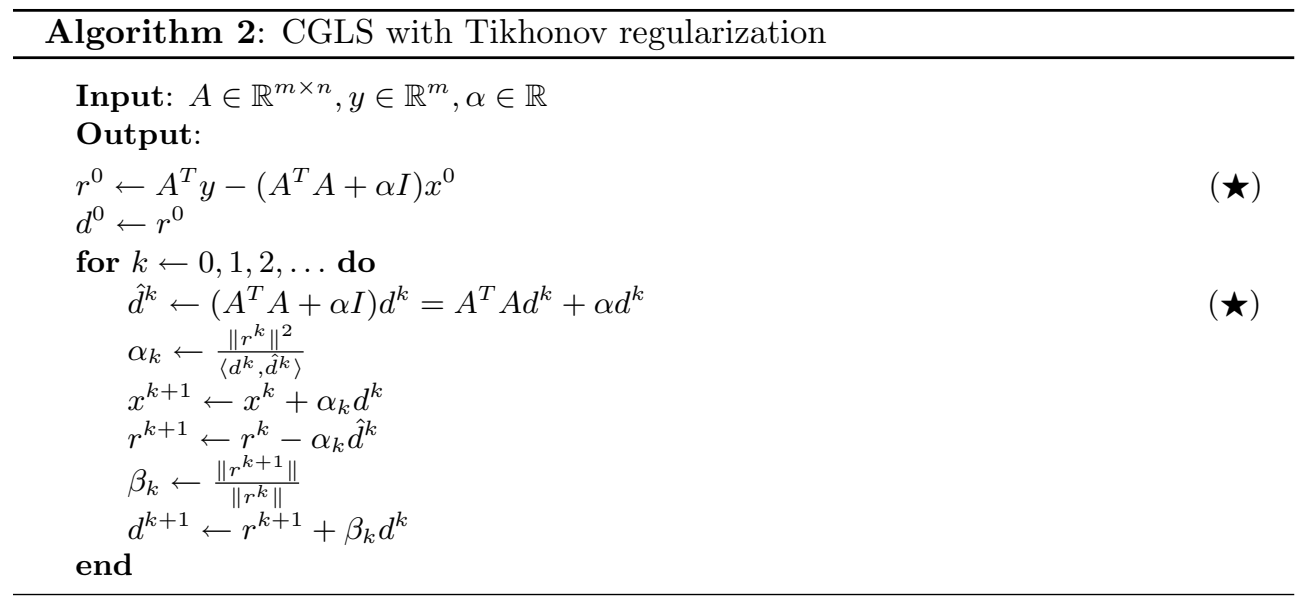

used incorporate a projection and a backprojection operator. These operators are applied line by line using classical ART or image by image using simultaneous ART (SART) (see [13]). The most time-consuming parts of a solver are the projection operations. Using a method that utilizes the system matrix $A$ only in products of the form $A x$ and $A^{T} y$, respectively, result in algorithms that do not need to have access to single matrix elements. Thus the projection and backprojection operators can be encapsulated and parallelized line by line. These properties make the operators appropriate for the efficient implementation on modern graphics hardware. Due to the ill-posed projection equation we solve the regularized problem

$$
\|A x-b\|^{2}+\alpha\|x\|^{2} \rightarrow \min _{x}
$$

with a fixed $\alpha>0$ using the conjugate gradient (CG) method. This kind of regularization, using the penalty factor $\|x\|^{2}$, is well known as standard Tikhonov regularization (see $[5,6])$. Equivalent to $(3.2)$ is the normal equation

$$
\left(A^{T} A+\alpha I\right) x=A^{T} b
$$

with the unique solution

$$
x_{\alpha}=\left(A^{T} A+\alpha I\right)^{-1} A^{T} b .
$$

The regularization parameter $\alpha$ has to be chosen a priori. It controls the proportion of noise that should be filtered out of the solution. An overview of strategies to automatically determine $\alpha$ can be found in [4, 25].

We use a variant of the CG method which allows the projection and backprojection steps to immediately follow each other (see Algorithm 2). This guarantees that both projections can run purely on the GPU without using external memory. The GPU-specific details will be discussed in the next section. The parts of the CG algorithm that run on the GPU are marked by $(\star)$ in Algorithm 2. It is well known that this variant of the CG method shows instabilities at high iteration numbers $[19,2]$. On the other hand the results (section 5) show that for image reconstruction only a few (mostly $\leq 10)$ iterations suffice to reach the desired reconstruction quality, and thus no disadvantage may be expected (see Figure 5.2, left-hand side). This holds 
especially for reconstructions from very few images (see Figure 5.2, right-hand side). We observed that other CG variants achieve comparable results in significantly higher computation times.

Given an encapsulated projection-backprojection step on the GPU the solver may easily be exchanged. Hence, we can benefit from nonlinear regularizations, e.g., total variation (TV) [25]. We solved the resulting minimization problem

$$
\|A x-b\|^{2}+\beta \operatorname{TV}(x) \rightarrow \min
$$

using a two-point step size gradient method [1] (see Figure 5.6).

4. GPU implementation. As shown in the preceding section, the projection algorithm as well as the method for solving the system of equations have been carefully designed and chosen to enable an efficient implementation on modern graphics hardware. The GPU can be seen as a stream processor, meaning that it operates on a stream of data. A small program called the kernel is working with these data. The kernel is executed once for each element of the data stream. It can read data from the stream but write data only at the actual position. Many of these kernels can be executed independently.

A GPU is designed to execute simple calculations for many pixels simultaneously. It has a parallel single-instruction multiple-data architecture based on a limited instruction set. Modern GPUs contain user-programmable vertex and fragment shaders. Thereby the vertex shaders supply the fragment shaders with linear interpolated values. A vertex shader performs complex calculations whose results change only linearly for each pixel. A fragment shader containing the kernel program performs on the output of the vertex shader. It is executed exactly once for each pixel.

To use GPUs as general-purpose parallel computing devices, standard data types are required. In this work we represent data streams as $2 \mathrm{D}$ textures which are completely transferred into the graphics memory before starting any calculations. The vertex shader can be used to calculate all values that are affine linear with respect to the coordinates of the pixels. These values can be obtained by a simple barycentric interpolation of the coordinates of the pixels which can be accomplished very efficiently by the graphics hardware. Summing up, the kernel function has to be built using the following scheme:

$$
\operatorname{OutputStream}(i, j)=\operatorname{Kernel}(i, j, \text { InputStream }) .
$$

Due to this scheme, the kernel function can be executed in parallel on the complete grid $(i, j), 1 \leq i, j \leq n$. This operation is mapped onto a single rendering process and therefore is completely controlled by the GPU.

In the following we detail the implementation of the matrix vector products $A x$ and $A^{T} y$ on the GPU as part of the CG method used in Algorithm 2. The decomposition of a complete projection-backprojection procedure into elementary subroutines is depicted in Algorithm 3.

We slice the volume in the $z$ direction, resulting in a stack of 2D textures stored in the graphics memory. The X-ray images are also represented as a list of $2 \mathrm{D}$ textures. The textures representing the images as well as the volume form the input and output streams of our kernel operations described in the following. According to (2.3) the projection onto an X-ray image is realized in layers. Thereby each rendering procedure uses an additional texture storing the accumulated values. This approach to dealing with the read-only and write-only problem of the graphics hardware architecture, 


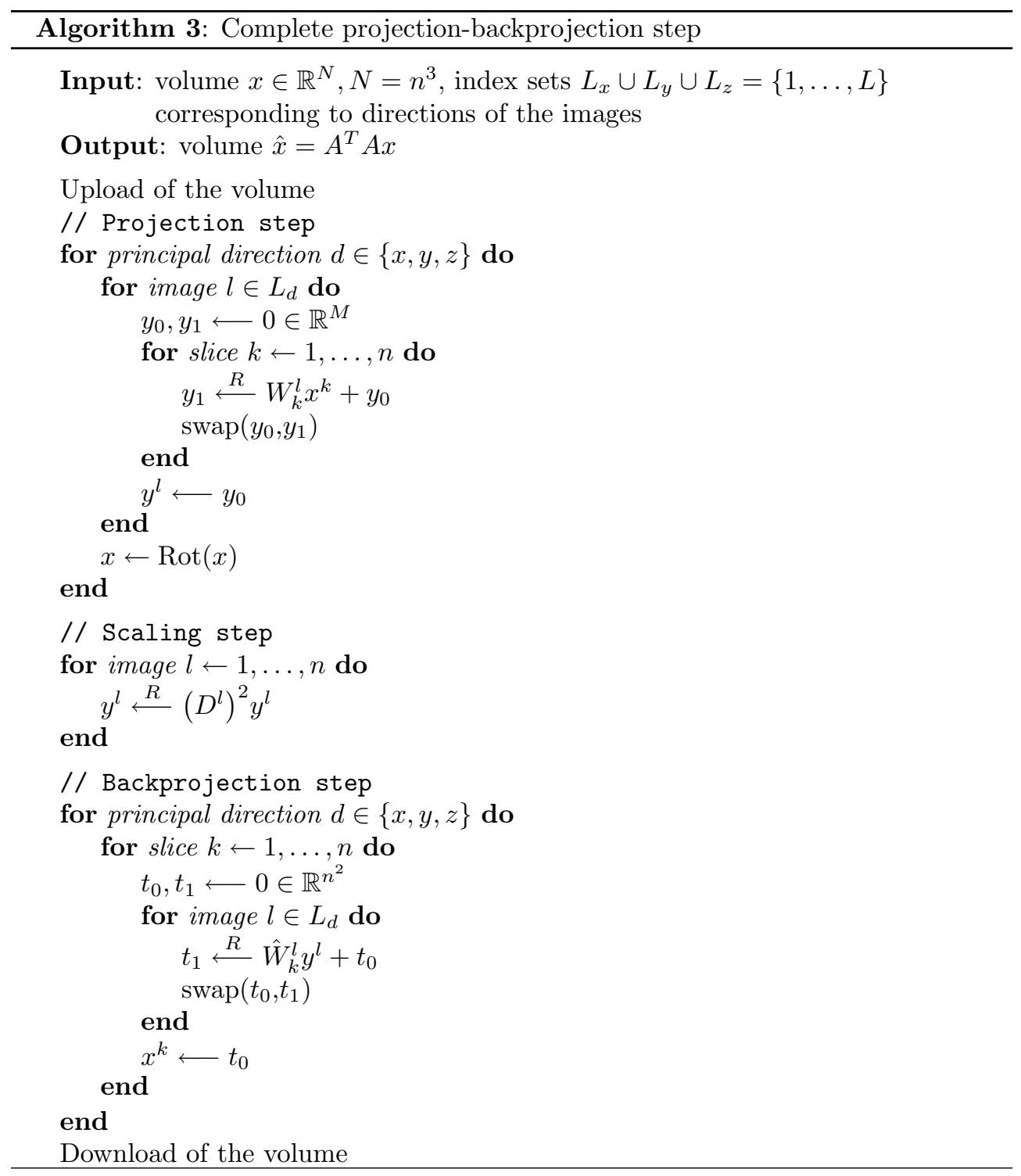

respectively, is known as the ping-pong technique. Projection as well as backprojection are the core of Algorithm 3, where they are marked by an $R$. To prevent distortions the projections are split into three groups. Thereby the volume is sliced into a stack of $2 \mathrm{D}$ textures in that direction minimizing the angle to the image normal. Each image belongs to one of the groups $L_{x} \cup L_{y} \cup L_{z}=\{1, \ldots, L\}$ depending on its surface normal. Switching between the different groups can be achieved by permuting the elements of the volume stack. This permutation in fact is equivalent to rotating the coordinate system containing the volume by $\frac{2 \pi}{3}$ about the axis $(1,1,1)^{T}$. The first rotation changes the direction of the $2 \mathrm{D}$ texture stack from $z$ to $x$, the next one from $x$ to $y$ :

$$
(\operatorname{Rot} v)(i, j, k):=v(k, j, i), \quad 1 \leq i, j, k \leq n .
$$




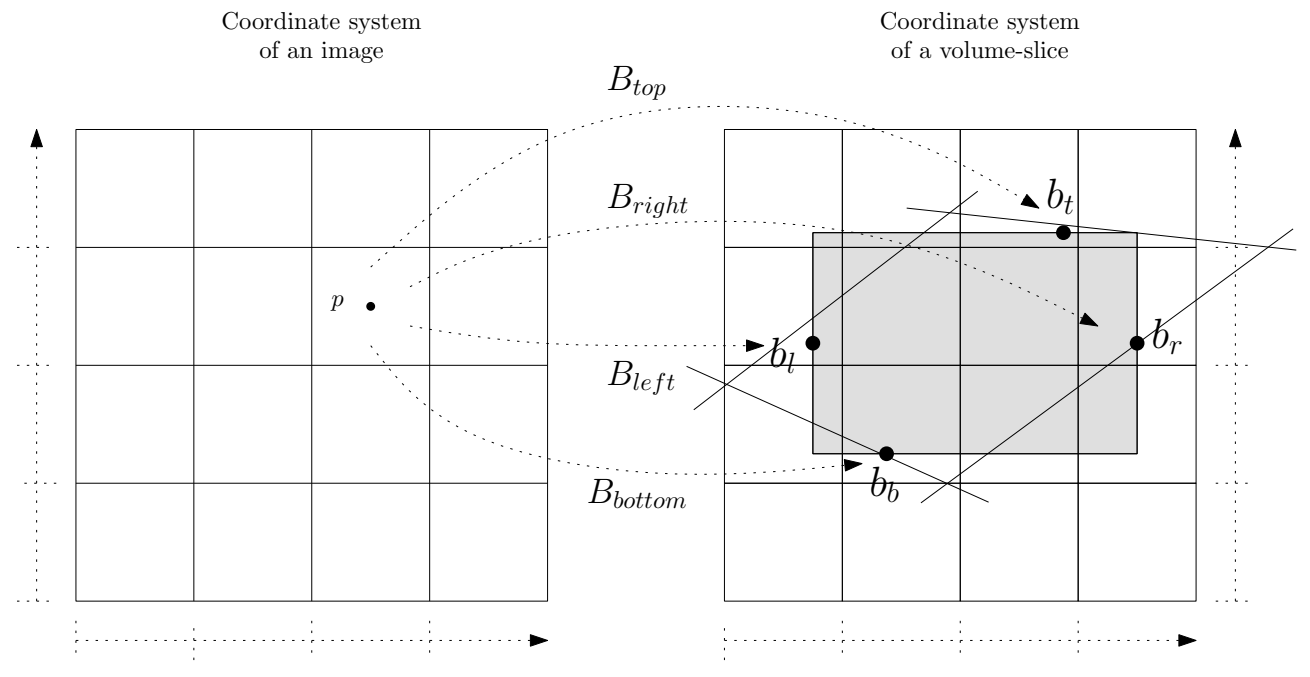

FIG. 4.1. The vertex shader supplies four varying variables $b_{l}, b_{b}, b_{r}, b_{t}$ corresponding to the backprojected boundaries of the pixel p; cf. Figure 2.2 and (4.2) for the definition of the mappings $B_{\text {left }}, \ldots, B_{\text {top }}$.

These rotations also completely take place on the graphics board and therefore do not produce any memory traffic between GPU and CPU [28]. Note, however, that they require memory for two volumes simultaneously in total.

Projection kernel. The major task of the projection kernel is the determination of the boundaries of each pixel of the X-ray image backprojected into the volume according to Figure 2.2 as well as the evaluation of the linear combination of the voxel values with the overlapping ratios $\frac{o_{i j}}{o_{i}^{k}}$ as weighting according to (2.1). Thereby the boundaries of the pixels are backprojected by the vertex processor. In this process for each 2D coordinate of a pixel, four 2D coordinates of a projected voxel have to be calculated: $b_{l}, b_{b}, b_{r}, b_{t}$. These four coordinates are given by four projective mappings $B_{\text {left }}, B_{\text {bottom }}, B_{\text {right }}, B_{\text {top }}$ of the coordinates of the current pixel midpoint $p$ which have to be passed to the vertex processor:

$$
\begin{aligned}
b_{l} & =B_{\text {left }}(p)=\mathcal{B}\left(p-\frac{1}{2} d_{x}\right), & b_{r} & =B_{\text {right }}(p)=\mathcal{B}\left(p+\frac{1}{2} d_{x}\right), \\
b_{b} & =B_{\text {bottom }}(p)=\mathcal{B}\left(p-\frac{1}{2} d_{y}\right), & b_{t} & =B_{\text {top }}(p)=\mathcal{B}\left(p+\frac{1}{2} d_{y}\right) .
\end{aligned}
$$

Thereby the projective mapping $\mathcal{B} \in \mathbb{R}^{3 \times 3}$ backprojects a point onto the image plane into the volume layer according to the projection geometry. The vectors $d_{x}$ and $d_{y}$ are both orthonormal and via the conditions

$$
\left(b_{r}\right)_{y}=\left(b_{l}\right)_{y} \quad \text { and } \quad\left(b_{t}\right)_{y}>\left(b_{b}\right)_{y}
$$

uniquely defined.

The calculated coordinates are related to texture coordinates within the image textures as well as textures representing a volume layer. The task of the vertex shader is illustrated in Figure 4.1. A visualization of the approximation process is given in Figure 4.2. Shown is the resulting rectangle pattern as the consequence of backprojecting and approximating the whole pixel grid of an image. The actual backprojection of the pixel boundaries is thereby accomplished by the interpolation 


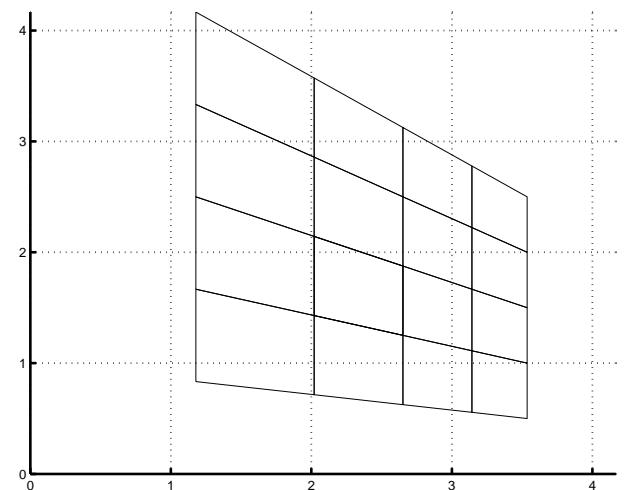

(a)

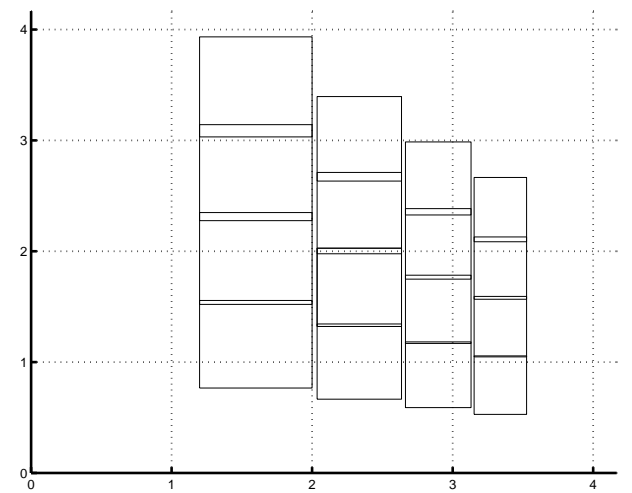

(b)

FIG. 4.2. Simulation of the rectangle approximation process as implemented. (a) shows the exact backprojection of the pixel grid onto the midplane of a volume layer. (b) shows the resulting approximation rectangles. The given coordinate system is exactly that of the midplane of the volume layer and thus the same as on the right-hand side of Figure 4.1.

hardware of the GPU. In contrast to nearest-neighbor or bilinear interpolation, at which only one or, respectively, four adjacent voxels of a volume layer contribute to the sampled value, overlap-based interpolation involves every voxel which overlaps the computed rectangle. As a consequence of backprojecting the pixel area, the spatial size of a pixel has an impact on the number of voxels contributing to the projection value, whereas bilinear interpolation only traces a ray through the volume, ignoring the pixel size.

Backprojection. Backprojection of an image is accomplished separately for each combination of the image with a volume layer. As in the projection step the volume is sliced into the image sets $L_{x}, L_{y}, L_{z}$ according to (4.1). Up to a scale factor the kernel of the backprojection step is the same as the kernel used in the projection step. The backprojection kernel projects the voxel boundaries onto the current image and accumulates the pixel values according to the overlapping ratio $\frac{\hat{o}_{j i}}{s}$ from $(2.2)$. Because this ratio is measured in the coordinate system of the image we obtain a pixel size of $s=1$.

5. Results. In the following we present reconstructions and timings of simulated as well as real-world data. In Table 5.1 a list of reconstruction times of the GPU implementation are given. The speedup factors between the identical CPU implementation on a single core and the new GPU approach ranges between 25 and 250.

In our simulations we studied different projection methods, noise levels and regularization techniques, iteration counts, and the effects of misaligned data. We particularly concentrated on the latter since the real datasets presented in Figures 5.5 and 5.6 de facto are based on slightly misaligned projections due to registration errors.

5.1. Simulated data. We validate our approach with a Shepp-Logan phantom, from which we produce varying numbers of projection images. The arrangement of the synthetic projection images is always uniformly distributed over a single circular orbit. In Figure 5.1 we concentrate on quality improvement provided by the overlap-based projection algorithm in comparison to well-established nearest-neighbor and bilinear interpolation methods. We show reconstructions from a varying number of projections and different noise levels. The increasing reconstruction quality and reduced noise is clearly visible, especially for very few projections, on which we concentrate in this work. 
TABLE 5.1

Reconstruction time for the simulated Shepp-Logan phantom displayed in Figure 5.1. A comparison between CPU (Intel Core 2 2.13GHz, SingleCore usage) and GPU (NVIDIA GeForce 8800 $G T X 768 M B)$ time is presented for one iteration performed.

\begin{tabular}{cc|ccc|ccc}
\hline \hline & & \multicolumn{3}{|c|}{10 projection images } & \multicolumn{3}{c}{50 projection images } \\
\hline $\begin{array}{c}\text { Volume } \\
\text { size }\end{array}$ & $\begin{array}{c}\text { Projection } \\
\text { size }\end{array}$ & \multicolumn{2}{|c}{ Time } & CPU & GPU & fpeedup & \multicolumn{2}{c}{ Time } & Speedup \\
factor & CPU & GPU & factor $(\approx)$ \\
\hline $64^{3}$ & $128 \times 128$ & $10.1 \mathrm{~s}$ & $0.4 \mathrm{~s}$ & 25 & $50.1 \mathrm{~s}$ & $1.2 \mathrm{~s}$ & 40 \\
$64^{3}$ & $256 \times 256$ & $38.9 \mathrm{~s}$ & $0.7 \mathrm{~s}$ & 55 & $194.3 \mathrm{~s}$ & $2.4 \mathrm{~s}$ & 80 \\
$128^{3}$ & $256 \times 256$ & $79.1 \mathrm{~s}$ & $1.8 \mathrm{~s}$ & 40 & $394.5 \mathrm{~s}$ & $4.0 \mathrm{~s}$ & 100 \\
$128^{3}$ & $512 \times 512$ & $306.0 \mathrm{~s}$ & $2.9 \mathrm{~s}$ & 105 & $1524.8 \mathrm{~s}$ & $9.1 \mathrm{~s}$ & 165 \\
$256^{3}$ & $512 \times 512$ & $626.3 \mathrm{~s}$ & $11.3 \mathrm{~s}$ & 55 & $3121.6 \mathrm{~s}$ & $19.7 \mathrm{~s}$ & 160 \\
$256^{3}$ & $1024 \times 1024$ & $2420.9 \mathrm{~s}$ & $17.0 \mathrm{~s}$ & 140 & $12080.4 \mathrm{~s}$ & $48.1 \mathrm{~s}$ & 250 \\
$320^{3}$ & $512 \times 512$ & $800.3 \mathrm{~s}$ & $20.8 \mathrm{~s}$ & 40 & $3987.1 \mathrm{~s}$ & $30.8 \mathrm{~s}$ & 130 \\
$320^{3}$ & $1024 \times 1024$ & $3034.0 \mathrm{~s}$ & $26.9 \mathrm{~s}$ & 110 & $15184.4 \mathrm{~s}$ & $61.0 \mathrm{~s}$ & 250 \\
\hline \hline
\end{tabular}

As each iteration step is time-consuming, the iterative process should be stopped after a few steps, but also should reach the best possible solution until then. Considering the convergence of the iterative solving process, within the simulated scenario we can compare the solution of each iteration step against the original data. The left-hand side of Figure 5.2 shows that only a few iterations are sufficient to provide an adequate solution for a fixed number of projection images. Measured values include the residual norm $\left\|A^{T} b-A^{T} A x_{k}\right\|$ of the normal equation, the relative error $\left\|x-x_{k}\right\| /\|x\|$, and the norm $\left\|x_{k}\right\|$ of the $k$ th iteration. On the right side of Figure 5.2 the relative error according to the number of projections is depicted. Our projection algorithm used in combination with the CG method can reconstruct the Shepp-Logan phantom within a relative error under $1 \%$ beyond 200 projections. For reconstructions from few (32) projection images, the relative error ranges between 0.3 and 0.6 in an error-free scenario, which corresponds to the visual impression from Figure 5.1.

A second source of errors which especially occurs in image reconstruction from few images and which is often neglected in simulations are perturbations of the geometric setup. Such misaligned projection data can be induced by the registration method to arrange the projections properly or by hardware inaccuracies and should be handled by the solver (see also section 5.2 about reconstructions from real data). To simulate small errors in the geometry setting, in Figure 5.3 we perturb the projections of the Shepp-Logan phantom by randomly varying each projection origin in the $x, y$, and $z$ directions within a multiple of the pixel spacing (factor 15 in Figures 5.3 and 5.4). The perturbation results in an unstable convergence of the solver, and thus leads to meaningless reconstructions if not stopped after an appropriate number of iterations. However, Tikhonov regularization with appropriate regularization parameter $\alpha$ delivers also a stable solution over many iterations even if the projection data include geometric perturbations. If an estimation of the iteration number with minimal error is possible, the reconstruction process may be interrupted alternatively without using Tikhonov regularization. As a second regularization technique we used total variation as the penalty function to handle geometrically perturbed projection data. Figure 5.4 shows reconstructions based on total variation minimization from the same perturbed data as in Figure 5.3. In comparison with the CG reconstruction in Figure 5.3(a) at iteration number 6 the leftmost reconstruction in Figure 5.4 comprises an error reduction of about $10 \%$. Additionally, the total variation regularization results in a visually more homogeneous reconstructed object. 

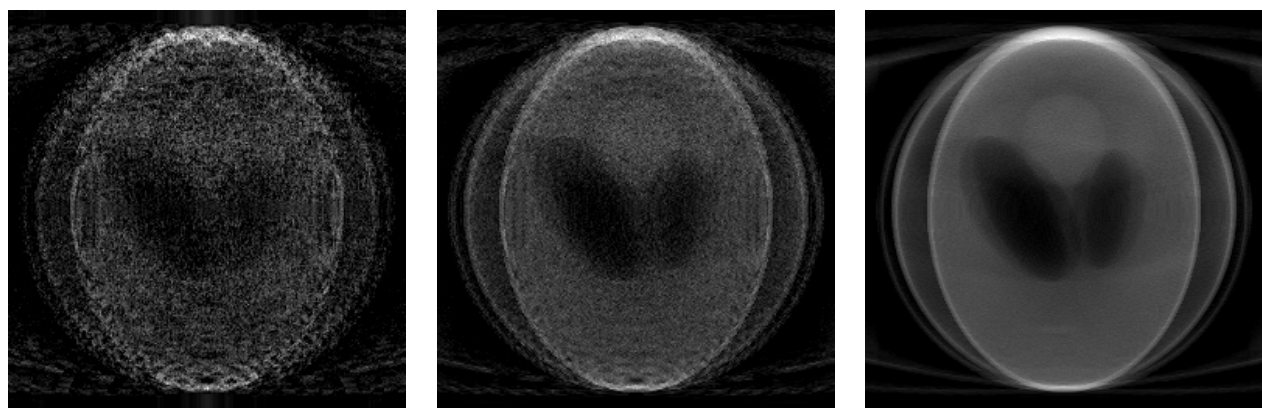

5 projections, noise level $2.5 \%$
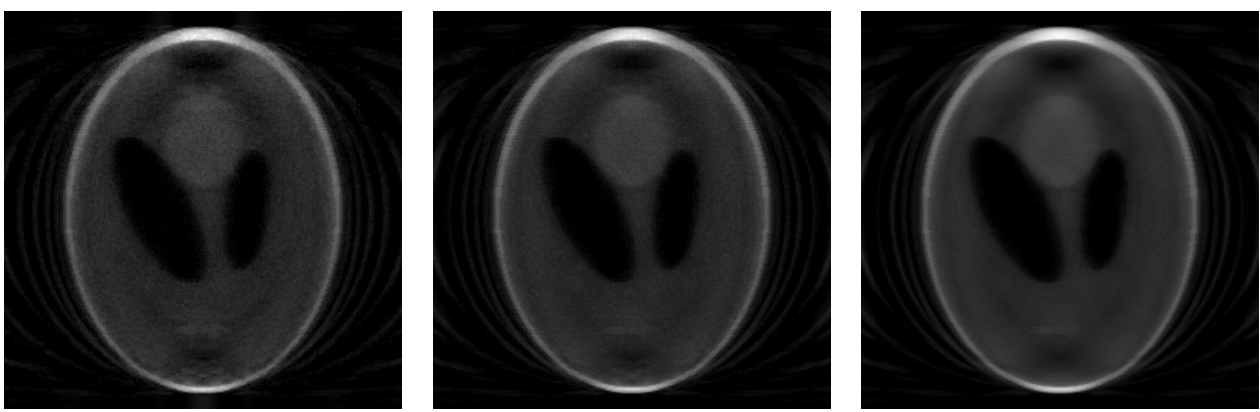

15 projections, noise level $2.5 \%$
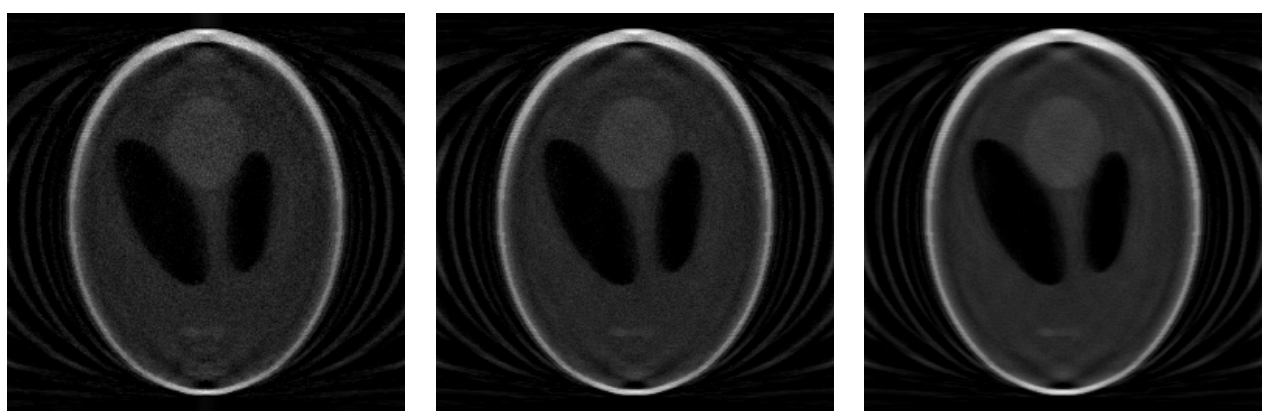

30 projections, noise level $5 \%$

FIG. 5.1. 3D reconstructions of a Shepp-Logan phantom: Comparison of nearest-neighbor (left column) and bilinear interpolation (middle column) to our projection algorithm (right column). Slices through the reconstruction volume of size $256^{3}$ after 10 iterations are shown. From top to bottom the number of projection images with size $512^{2}$ is increased gradually, as is the noise level.

5.2. Real data. Figures 5.5 and 5.6 show the reconstruction of a hard plaster specimen and a small song bird, respectively. The arrangement of the projections is visualized in Figure 1.1. Both series were registered with the reference sphere method [21]. We display raw reconstructions without postprocessing; only a manually determined opacity transfer function was applied to control which parts of the data are visible. Since we employ different X-ray source and detector systems (a commercial dental CCD sensor, a medical amorphous selenium detector, and an indirect digital system with a storage phosphor plate), no a priori information about the detector and its noise characteristic is included in our reconstruction process. 

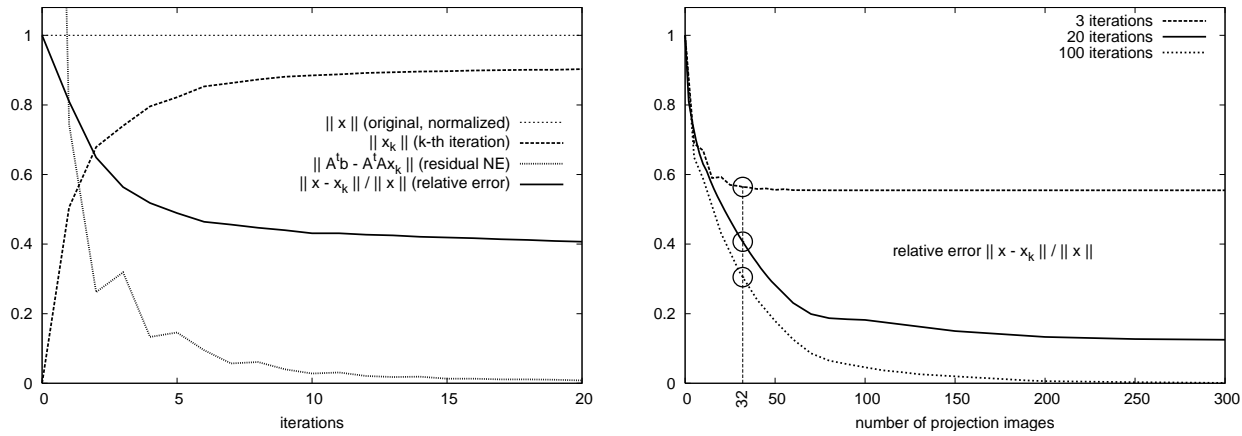

FIG. 5.2. Convergence for solving the linear system with the simulated, error-free Shepp-Logan phantom using the CG method with volume size $128^{3}$ and image size $512^{2}$. On the left-hand side the error characteristics of the first 20 iterations for 32 projections are displayed. On the right-hand side the number of projection images is varied for a constant number of 3, 20, and 100 iterations.

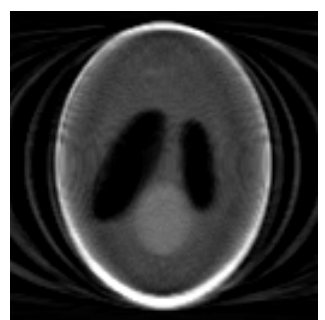

iteration 6

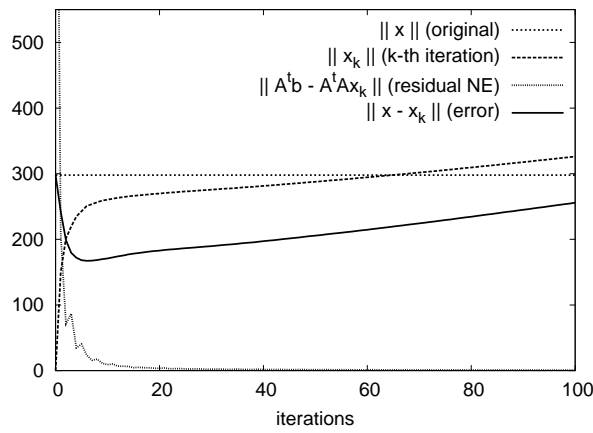

(a) geometrically perturbed data

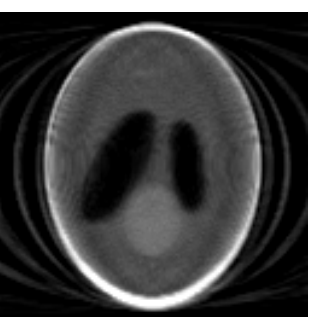

iteration 6

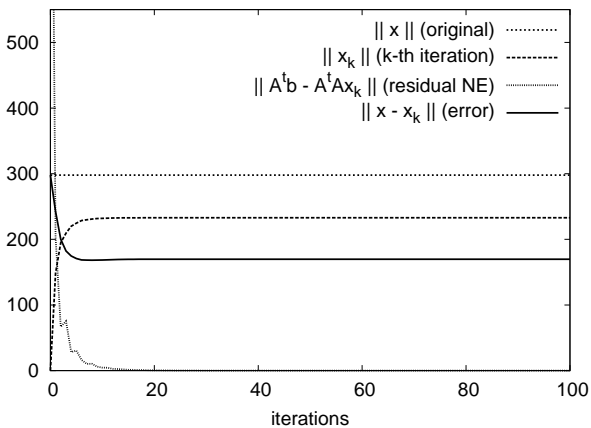

(b) geometrically perturbed, Tikhonov-regularized

FIG. 5.3. In (a) the convergence of a perturbed Shepp-Logan phantom reconstructed from 30 projection images using the $C G$ method is displayed. After reaching the minimal relative error at iteration number 6 the reconstruction diverges and shows significantly more artifacts. In (b) Tikhonov regularization with the constant regularization parameter $\alpha=0.1$ is applied. In doing so the reconstruction converges against a stable solution already after few iterations.

For the reconstructions (b), (c) in Figure 5.5 the CG method without regularization was applied, and we stopped the $\mathrm{CG}$ reconstruction process using the relative residual norm $\left\|A^{T} b-A^{T} A x_{k}\right\| /\left\|A^{T} b\right\|$ of the normal equation. As a good termination condition for our real datasets, a level of $1 \%$ can be assumed and results in iterations between 5 and 15 . In the nonlinear process of total variation minimization [1] we used a fixed number of 30 iterations.

Figure 5.6 compares a reconstruction without regularization (b), (d) and a reconstruction with total variation regularization (c), (e), (f). When computing the 


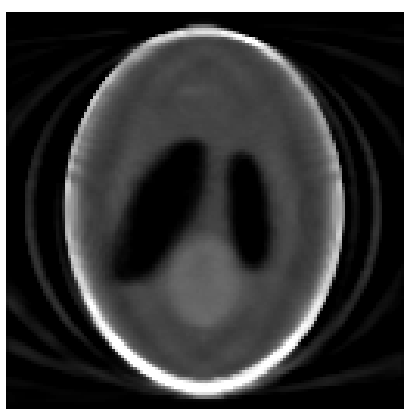

$\beta=0.01$

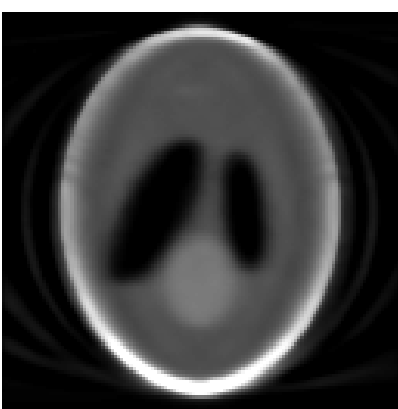

$\beta=0.05$

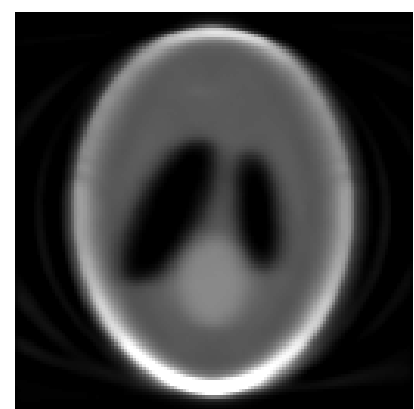

$\beta=0.1$

FIG. 5.4. Reconstructions from geometrically perturbed data based on total variation minimization. Thereby different regularization parameters $\beta$ are chosen showing an increasing homogeneity of the solution. See also Figure 5.3 to compare with a Tikhonov regularized reconstruction from the same perturbed data.

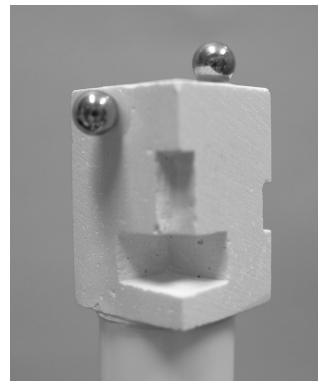

(a)

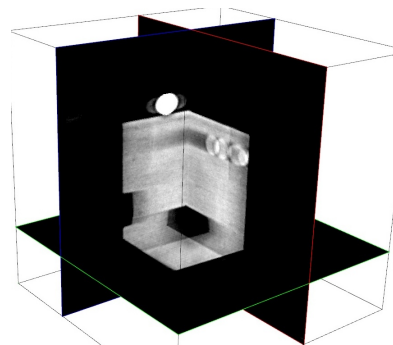

(b)

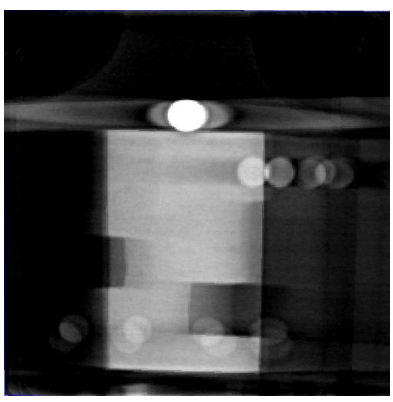

(c)

FiG. 5.5. CG reconstruction (b), (c) of a hard plaster specimen (a) exposed on a dental CCD sensor in twelve varying positions. The shadows of the reference spheres and the artifacts around them are clearly visible; however, no attempt to suppress them or other artifacts has been carried out.

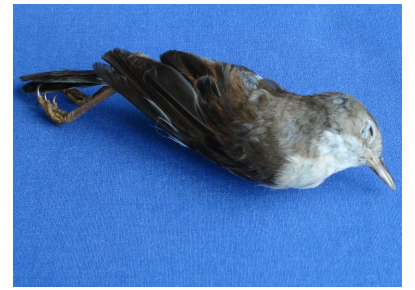

(a)

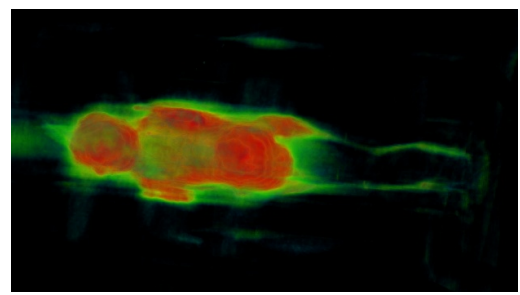

(d)

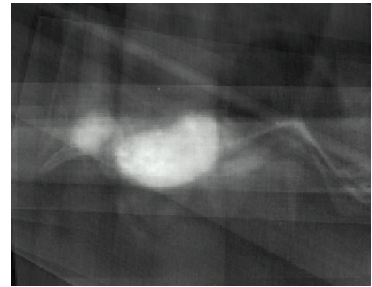

(b)

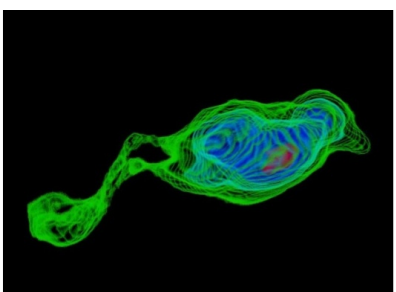

(e)

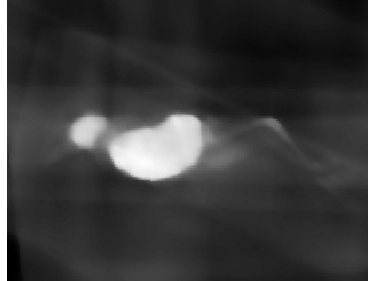

(c)

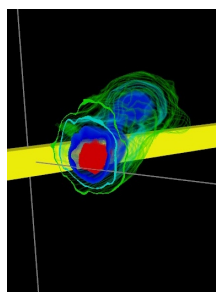

(f)

FIG. 5.6. Reconstruction using no regularization (b), (d) versus total variation regularization (c), (e), (f) from eight projections of a small song bird (genus Sylvia) shown in (a) on a storage phosphor plate. 
reconstructions of the bird, we manually selected the value for the regularization parameter according to visual judgment. Total variation regularization enhances reconstruction quality in few-view reconstructions, since the object to be reconstructed is reduced to few homogeneous regions and noise is suppressed.

6. Discussion and conclusion. In recent years, the research community has directed increasing attention towards reconstruction of 3D structures from few radiographic projections, which requires drastically reduced radiation dosages compared to established 3D techniques. Few-view reconstruction is very flexible with respect to input data, geometric properties of the projections, and size of the object under investigation. A good example of a few-view limited-angle reconstruction technique currently in the prototype stage but with high potential is breast tomosynthesis $[27,30]$. From a mathematical point of view few-view reconstruction represents a severely ill-posed inverse problem. Hence, it is computationally challenging. Our approach presented here particularly suits arbitrary imaging geometries; i.e., the acquisition of the projections is not constrained at all. There has not been much research using arbitrary acquisition geometries; however, if one aims to use existing radiographic projection devices, they represent the input data one might expect to see. Yet arbitrary geometries make the reconstruction problem even more challenging, since no common geometric pattern can be established between the projections. We present a method to produce artifact-reducing few-view reconstructions using the GPU. Implementation on the GPU due to its massively parallelized architecture drastically reduces the time required for the projection and backprojection steps during the 3D reconstruction process $[13,28]$. Thereby, reconstruction time on an up-to-date GPU interfaced with an off-the-shelf personal computer is reduced to minutes. Volume sizes of up to $320^{3}$ can be reconstructed within about five minutes. Here the volume size is limited by the graphics memory available. By extending an approach introduced by [3] to arbitrary projection geometries, the volume a voxel shares with the "ray" backprojected from the detector pixel is approximated with high accuracy. This is one of the major contributions of this work, since inaccurate approximations result in typical aliasing artifacts in the reconstruction. As demonstrated, our method prevents aliasing artifacts almost completely. GPU implementation makes the method fast enough for real-world applications. The system of equations is solved iteratively by means of a conjugate gradient algorithm modified for the GPU. Apart from linear Tikhonov regularization, we also implemented a nonlinear method of total variation minimization [1]. Future work will include a thorough evaluation of its performance when compared to Tikhonov regularization or other methods. In fact, further investigation of the few-view limited-angle reconstruction problem will focus on efficient regularization, including statistical approaches based on Bayesian inversion [24, 10, 11, 18]. The capability of the latter to further reduce problem-related reconstruction artifacts has been demonstrated already [10, 11, 18].

In conclusion, we present an approach to efficiently implement an accurate algorithm for few-view limited-angle 3D reconstruction for projections acquired at arbitrary geometries. Flexibility with respect to object size, radiographic device, or acquisition geometry and dose-reduction capability are the main advantages of fewview reconstruction approaches over established 3D techniques such as CT or conebeam CT. We expect that these advantages, combined with the upcoming advances in hardware technology, will further increase research interest in few-view reconstruction techniques. Our approach may be one step along the way to establishing such techniques in industrial nondestructive testing, medical, or security technology. 
Acknowledgment. We gratefully acknowledge our former student Min Sung Kim from the Dental School of the Johannes Gutenberg-University Mainz for preparing and providing the hard plaster series.

\section{REFERENCES}

[1] J. Barzilai And J. M. Borwein, Two-point step size gradient methods, J. Numer. Anal., 8 (1988), pp. 141-148.

[2] A. BJörck, Numerical Methods for Least Squares Problems, SIAM, Philadelphia, 1996.

[3] B. De Man And S. Basu, Distance-driven projection and backprojection in three dimensions, Phys. Med. Biol., 49 (2004), pp. 2463-2475.

[4] G. H. Golub and U. von MatT, Tikhonov regularization for large scale problems, in Proceedings of the Workshop on Scientific Computing, G. H. Golub, S. H. Lui, F. Luk, and R. J. Plemmons, eds., Springer-Verlag, New York, 1997, pp. 3-26.

[5] C. W. Groetsch, The Theory of Tikhonov Regularization for Fredholm Equations of the First Kind, Pitman, Boston, 1984.

[6] M. Hanke And P. C. Hansen, Regularization methods for large-scale problems, Surv. Math. Indust., 3 (1993), pp. 253-315.

[7] M. KACHELRIESS AND M. KNAUP, Hyperfast parallel-beam and cone-beam backprojection using the cell general purpose hardware, Med. Phys., 34 (2007), pp. 1474-1485.

[8] J. Kaipio And E. Somersalo, Statistical and Computational Inverse Problems, Appl. Math. Sci., Springer-Verlag, New York, 2005.

[9] A. C. KaK And M. Slaney, Principles of Computerized Tomographic Imaging, IEEE Press, New York, 1988; reprinted as Classics Appl. Math. 33 by SIAM, Philadelphia, 2001.

[10] V. Kolehmainen, A. Vanne, S. Siltanen, S. JÄrvenpä̈̈, J. Kaipio, M. LAssas, And M. KALKe, Parallelized Bayesian inversion for three-dimensional dental $x$-ray imaging, IEEE Trans. Med. Imaging, 25 (2006), pp. 218-228.

[11] V. Kolehmainen, A. Vanne, S. Siltanen, S. Järvenpä̈̈, J. Kaipio, M. Lassas, And M. KALKE, Bayesian inversion method for 3-d dental x-ray imaging, Elektrotechnik \& Informationstechnik, 124 (2007), pp. 248-253.

[12] C. LeHR AND C. E. LiedtKe, $3 d$ reconstruction of volume defects from few $x$-ray images, Lecture Notes in Comput. Sci. 1689, Springer-Verlag, London, 1999, pp. 275-284.

[13] K. Mueller, Fast and Accurate Three-Dimensional Reconstruction from Cone-Beam Projection Data Using Algebraic Methods, Ph.D. thesis, Ohio State University, Columbus, OH, 1998.

[14] K. Mueller, F. Xu, And N. Neophytos, Why do commodity graphics hardware boards (GPUs) work so well for acceleration of computed tomography?, Proc. SPIE, 6498 (2007), pp. 1-12.

[15] K. Mueller And R. YAGel, Rapid 3-d cone-beam reconstruction with the simultaneous algebraic reconstruction technique (SART) using 2-d texture mapping hardware, IEEE Trans. Med. Imaging, 19 (2000), pp. 1227-1237.

[16] F. NATTerer, Numerical methods in tomography, Acta Numerica, 8 (1999), pp. $107-141$.

[17] N. Neophytos, F. Xu, And K. Mueller, Hardware acceleration vs. algorithmic acceleration: Can GPU-based processing beat complexity optimization for CT?, Proc. SPIE, 6510 (2007), pp. $1-9$.

[18] K. Nimimäki, S. Siltanen, And V. Kolehmainen, Bayesian multiresolution method for local tomography in dental x-ray imaging, Phys. Med. Biol., 52 (2007), pp. 6663-6678.

[19] C. C. Paige And M. A. Saunders, LSQR: An algorithm for sparse linear equations and sparse least squares, ACM Trans. Math. Software, 8 (1982), pp. 43-71.

[20] M. Rantala, S. Vanska, S. Järvenpä̈, M. Kalke, M. Lassas, J. Moberg, and S. SiltaNEN, Wavelet-based reconstruction for limited-angle $x$-ray tomography, IEEE Trans. Med. Imaging, 25 (2006), pp. 210-217.

[21] R. Schulze, U. Heil, O. Weinheimer, D. Gross, D. D. Bruellmann, E. Thomas, U. SchwaNECKE, AND E. SCHOEMER, Accurate registration of random radiographic projections based on three spherical references for the purpose of few-view $3 d$ reconstruction, Med. Phys., 35 (2008), pp. 546-555.

[22] G. C. Sharp, N. Kanasamy, H. Singh, and M. Folkert, GPU-based streaming architectures for fast cone-beam CT image reconstruction and demons deformable registration, Phys. Med. Biol., 52 (2007), pp. 5771-5783.

[23] E. Y. Sidky, C.-M. KAO, AND X. PAN, Accurate image reconstruction from few-views and limited-angle data in divergent-beam CT, J. X-Ray Sci. Technol., 14 (2006), pp. 119-139. 
[24] S. Siltanen, V. Kolehmainen, S. JärvenpäÄ, J. P. Kaipio, P. Koistinen, M. Lassas, J. Pirttilä, AND E. Somersalo, Statistical inversion for medical x-ray tomography with few radiographs: I. General theory, Phys. Med. Biol., 48 (2003), pp. 1437-1463.

[25] C. R. Vogel, Computational Methods for Inverse Problems, Frontiers Appl. Math. 23, SIAM, Philadelphia, 2002.

[26] R. L. Webber, R. A. Horton, D. A. Tyndall, and J. B. Ludlow, Tuned-aperture computed tomography (TACT). Theory and application for three-dimensional dento-alveolar imaging, Dentomaxillofacial Radiology, 26 (1997), pp. 53-62.

[27] T. Wu, R. H. Moore, E. A. Rafferty, and D. B. Kopans, A comparison of reconstruction algorithms for breast tomosynthesis, Med. Phys., 31 (2004), pp. 2636-2647.

[28] F. XU AND K. Mueller, Accelerating popular tomographic reconstruction algorithms on commodity PC graphics hardware, IEEE Trans. Nuclear Sci., 52 (2005), pp. 654-663.

[29] F. XU AND K. Mueller, Real-time $3 d$ computed tomographic reconstruction using commodity graphics hardware, Phys. Med. Biol., 52 (2007), pp. 3405-3419.

[30] Y. G. Zhang, H.-P. Chan, B. Sahiner, J. Wei, M. M. Goodsitt, L. M. Hadjisski, J. Ge, AND C. ZHOU, A comparative study of limited-angle cone-beam reconstruction methods for breast tomosynthesis, Med. Phys., 33 (2006), pp. 3781-3795. 